\title{
On Flows of Bingham-Type Fluids with Threshold Slippage
}

\author{
Evgenii S. Baranovskii \\ Department of Applied Mathematics, Informatics and Mechanics, Voronezh State University, Voronezh, Russia
}

Correspondence should be addressed to Evgenii S. Baranovskii; esbaranovskii@gmail.com

Received 20 September 2017; Accepted 26 November 2017; Published 17 December 2017

Academic Editor: Luigi C. Berselli

Copyright (C) 2017 Evgenii S. Baranovskii. This is an open access article distributed under the Creative Commons Attribution License, which permits unrestricted use, distribution, and reproduction in any medium, provided the original work is properly cited.

We investigate a mathematical model describing 3D steady-state flows of Bingham-type fluids in a bounded domain under threshold-slip boundary conditions, which state that flows can slip over solid surfaces when the shear stresses reach a certain critical value. Using a variational inequalities approach, we suggest the weak formulation to this problem. We establish sufficient conditions for the existence of weak solutions and provide their energy estimates. Moreover, it is shown that the set of weak solutions is sequentially weakly closed in a suitable functional space.

\section{Introduction}

The statement that a fluid adheres to any solid boundary is one of the main tenets of classical fluid mechanics. However, careful experiments point to various possibilities for the behaviour of fluids at the interphase boundary. In particular, it is known that many non-Newtonian fluids slip over solid surfaces when the shear stresses reach a critical value. In order to describe slip effects, numerous mathematical models have been proposed (see, e.g., the short survey [1]).

In this article, we consider a model describing internal steady-state flows of a viscoplastic fluid of Bingham type $[2,3]$ in a bounded domain $\Omega \subset \mathbb{R}^{3}$ with locally Lipschitz boundary $\Gamma$ under a threshold-slip boundary condition [4] on a fixed subset $\Gamma_{0} \subset \Gamma$ and the no-slip condition on $\Gamma \backslash \Gamma_{0}$ :

$$
\begin{aligned}
& \rho \operatorname{div}(\mathbf{u} \otimes \mathbf{u})-\operatorname{div} \boldsymbol{\sigma}+\nabla \pi=\rho \mathbf{f} \quad \text { in } \Omega, \\
& \operatorname{div} \mathbf{u}=0 \quad \text { in } \Omega, \\
& \boldsymbol{\sigma}=\mu(|\mathbf{D}(\mathbf{u})|) \mathbf{D}(\mathbf{u})+g \frac{\mathbf{D}(\mathbf{u})}{|\mathbf{D}(\mathbf{u})|} \text { if }|\mathbf{D}(\mathbf{u})| \neq 0 \text { in } \Omega, \\
& |\boldsymbol{\sigma}| \leq g \quad \text { if }|\mathbf{D}(\mathbf{u})|=0 \text { in } \Omega, \\
& \mathbf{u} \cdot \mathbf{n}=0 \quad \text { on } \Gamma \\
& \left|(\boldsymbol{\sigma n})_{\tan }\right| \leq \omega \text { on } \Gamma_{0},
\end{aligned}
$$

$$
\begin{aligned}
& \left|(\boldsymbol{\sigma} \mathbf{n})_{\tan }\right|<\omega \Longrightarrow \mathbf{u}_{\tan }=\mathbf{0} \text { on } \Gamma_{0}, \\
& \left|(\boldsymbol{\sigma} \mathbf{n})_{\tan }\right|=\omega \Longrightarrow \mathbf{u}_{\tan } \uparrow \downarrow(\boldsymbol{\sigma} \mathbf{n})_{\tan } \text { on } \Gamma_{0}, \\
& \mathbf{u}=\mathbf{0} \text { on } \Gamma \backslash \Gamma_{0} .
\end{aligned}
$$

Here $\mathbf{u}$ is the velocity, $\pi$ is the pressure, $\boldsymbol{\sigma}$ is the deviatoric stress tensor, $\mathbf{f}$ is an external body force, $\mathbf{D}=\mathbf{D}(\mathbf{u})$ is the strain velocity tensor,

$$
D_{i j}=D_{i j}(\mathbf{u})=\frac{1}{2}\left(\frac{\partial u_{i}}{\partial x_{j}}+\frac{\partial u_{j}}{\partial x_{i}}\right),
$$

$\mu(|\mathbf{D}|)>0$ is the viscosity, $\rho$ is the constant density of the fluid, $g$ denotes the yield stress, $g: \Omega \rightarrow \mathbb{R}_{+}$, and $\omega$ is a critical value for start to slip along the boundary, $\omega: \Gamma_{0} \subset \Gamma \rightarrow \mathbb{R}_{+}$. For the sake of simplicity, we put in the sequel $\rho=1$.

The unknowns in systems (1)-(9) are the vector functions $\mathbf{u}, \boldsymbol{\sigma}$ and the function $\pi$, while all other quantities are assumed to be given.

Let us explain the tensor notation that we use in this article. Given a tensor $\mathbf{F}$, the vector $\operatorname{div} \mathbf{F}$ is defined by the formula

$$
(\operatorname{div} \mathbf{F})_{i}=\sum_{j=1}^{3} \frac{\partial F_{i j}}{\partial x_{j}}
$$


Given vectors $\mathbf{x}$ and $\mathbf{y}$, the tensor $\mathbf{x} \otimes \mathbf{y}$ is the tensor product defined by

$$
(\mathbf{x} \otimes \mathbf{y})_{i j}=x_{i} y_{j}
$$

We denote by $|\mathbf{v}|$ the Euclidean norm of a vector $\mathbf{v}$ and by $|\mathbf{E}|$ the Frobenius norm of a tensor $\mathbf{E}$ :

$$
\begin{aligned}
& |\mathbf{v}|^{2}=\mathbf{v} \cdot \mathbf{v}=\sum_{i=1}^{3} v_{i}^{2}, \\
& |\mathbf{E}|^{2}=\operatorname{trace}\left(\mathbf{E E}^{T}\right)=\sum_{i, j=1}^{3} E_{i j}^{2} .
\end{aligned}
$$

As usual, $\mathbf{n}$ denotes the unit outer normal to $\Gamma$ and $(\cdot)_{\tan }$ stands for the tangential component of a vector; that is,

$$
\mathbf{u}_{\tan }=\mathbf{u}-(\mathbf{u} \cdot \mathbf{n}) \mathbf{n} .
$$

The symbol $\uparrow \downarrow$ is used to denote oppositely directed vectors.

Remark 1 . Obviously, for $g \equiv 0$ and $\mu \equiv$ const, we recover the Navier-Stokes system with stick-slip boundary conditions. Such slip problem was studied in [4] (see also [5]). Note that system (6)-(8) is a special case of the following slip boundary condition [1]:

$$
\begin{gathered}
\left|(\mathbf{T n})_{\tan }\right| \leq \psi\left(\mathbf{x},\left|(\mathbf{T n})_{n}\right|\right), \\
(\mathbf{T n})_{\tan } \cdot \mathbf{u}_{\tan }=-\psi\left(\mathbf{x},\left|(\mathbf{T n})_{n}\right|\right)\left|\mathbf{u}_{\tan }\right|,
\end{gathered}
$$

where $\mathbf{T}=-\pi \mathbf{I}+\boldsymbol{\sigma}$ is the stress tensor, $(\mathbf{T n})_{n}=((\mathbf{T n}) \cdot \mathbf{n}) \mathbf{n}$, and $\psi: \Gamma_{0} \times \mathbb{R}_{+} \rightarrow \mathbb{R}_{+}$is a given function. Actually, if $\psi(\mathbf{x}, s)=$ $\omega(\mathbf{x})$ for any $(\mathbf{x}, s) \in \Gamma_{0} \times \mathbb{R}_{+}$, then it is easily shown that system (6) $-(8)$ is equivalent to (15).

The mathematical models of Bingham-type fluids are used to study the behaviour of materials such as paints, pastes, foams, suspensions, cements, and oils. Starting with the pioneering works by Mosolov and Miasnikov [6] and Duvaut and Lions [7], a large number of mathematicians have worked on the theoretical analysis of Bingham fluids and other similar viscoplastic media (see [8-23] and the references therein).

The novelty of the present paper is that it combines the use of the Bingham constitutive equations with threshold-slip boundary conditions and takes into account the dependence of the viscosity on the second invariant of the strain velocity tensor. It should be mentioned at this point that a nonlocal (regularized) friction problem for a class of non-Newtonian fluids has been investigated by Consiglieri [24] (see also [25]).

Let us state the main results of this paper. Following an approach adopted in $[4,7]$, we formulate the boundary-value problem (1)-(9) as a variational inequality for the unknown velocity field. Using some existence results for inequalities with pseudomonotone operators and convex functionals, which naturally arise in this slip problem, and the Krasnoselskii theorem on continuity of the Nemytskii operator [26], we establish sufficient conditions for the existence of weak solutions and derive their energy estimates. Also, it is shown that the set of weak solutions to problem (1)-(9) is sequentially weakly closed in a suitable functional space.

\section{Preliminaries}

In this section, we describe the necessary functional spaces and the main assumptions used in the paper.

We shall use the classical notation $L^{p}(\Omega)$ and $H^{s}(\Omega)=$ $W_{2}^{s}(\Omega)$ for the Lebesgue and Sobolev spaces, respectively. Bold face letters will denote functional spaces of vectors or tensors: $\mathbf{L}^{p}(\Omega)=L^{p}(\Omega)^{3}, \mathbf{H}^{s}(\Omega)=H^{s}(\Omega)^{3}$, and so forth.

Next, we set

$$
\begin{aligned}
& L_{+}^{p}(\Omega) \\
& :=\left\{\alpha \in L^{p}(\Omega): \alpha(\mathbf{x}) \geq 0 \text { for almost every } \mathbf{x} \in \Omega\right\}, \\
& \mathbf{Q}(\Omega) \\
& :=\left\{\mathbf{v} \in \mathbf{C}^{\infty}(\bar{\Omega}): \operatorname{div} \mathbf{v}=0,\left.\mathbf{v}\right|_{\Gamma} \cdot \mathbf{n}=0,\left.\mathbf{v}\right|_{\Gamma \backslash \Gamma_{0}}=\mathbf{0}\right\},
\end{aligned}
$$

$\mathbf{X}(\Omega)$

:= the closure of the set $\mathbf{Q}(\Omega)$ in the space $\mathbf{H}^{1}(\Omega)$.

We now recall an inequality of Korn's type.

Proposition 2. Let $a: \mathbf{H}^{1}(\Omega) \times \mathbf{H}^{1}(\Omega) \rightarrow \mathbb{R}$ be a continuous symmetric bilinear form such that $a(\mathbf{v}, \mathbf{v}) \geq 0$, for any $\mathbf{v} \epsilon$ $\mathbf{H}^{1}(\Omega)$, and it follows from the conditions

$$
\int_{\Omega}|\mathbf{D}(\mathbf{w})|^{2} d x=0, \quad a(\mathbf{w}, \mathbf{w})=0, \mathbf{w} \in \mathbf{H}^{1}(\Omega)
$$

that $\mathbf{w}=\mathbf{0}$. Then there exists a positive constant $C$ such that

$$
\|\mathbf{v}\|_{\mathbf{H}^{1}(\Omega)}^{2} \leq C\left(\int_{\Omega}|\mathbf{D}(\mathbf{v})|^{2} d x+a(\mathbf{v}, \mathbf{v})\right)
$$

for all $\mathbf{v} \in \mathbf{H}^{1}(\Omega)$.

The proof of this proposition is given in [27].

Suppose that the 2-dimensional Lebesgue measure of the set $\Gamma \backslash \Gamma_{0}$ is positive, then we can define the scalar product in $\mathbf{X}(\Omega)$ by the formula

$$
(\mathbf{v}, \mathbf{u})_{\mathbf{X}(\Omega)}=\int_{\Omega} \mathbf{D}(\mathbf{v}): \mathbf{D}(\mathbf{u}) d x,
$$

where $\mathbf{D}(\mathbf{v}): \mathbf{D}(\mathbf{u})$ denotes the scalar product of tensors $\mathbf{D}(\mathbf{v})$ and $\mathbf{D}(\mathbf{u})$ :

$$
\mathbf{D}(\mathbf{v}): \mathbf{D}(\mathbf{u})=\operatorname{trace}\left(\mathbf{D}(\mathbf{v}) \mathbf{D}(\mathbf{u})^{T}\right) .
$$

Setting

$$
a(\mathbf{v}, \mathbf{u}):=\int_{\Gamma \backslash \Gamma_{0}} \mathbf{v} \cdot \mathbf{u} d \Gamma \quad \forall \mathbf{v}, \mathbf{u} \in \mathbf{H}^{1}(\Omega)
$$

and applying Proposition 2, we infer that the norm

$$
\|\mathbf{v}\|_{\mathbf{X}(\Omega)}=(\mathbf{v}, \mathbf{v})_{\mathbf{X}(\Omega)}^{1 / 2}
$$

is equivalent to the norm induced from the Sobolev space $\mathbf{H}^{1}(\Omega)$. 
Recall that the restriction of a function $w \in H^{1}(\Omega)$ to $\Gamma$ is defined by the formula $\left.w\right|_{\Gamma}=\gamma_{0} w$, where $\gamma_{0}: H^{1}(\Omega) \rightarrow$ $H^{1 / 2}(\Gamma)$ is the trace operator (see [7]). 3.

By $\mathbb{M}_{\text {sym }}^{3 \times 3}$ denote the space symmetric matrices of size $3 \times$

In the sequel, we assume that the following conditions hold:

(i) for any matrices $\mathbf{A}, \mathbf{B} \in \mathbb{M}_{\text {sym }}^{3 \times 3}$, we have

$$
(\mu(|\mathbf{A}|) \mathbf{A}-\mu(|\mathbf{B}|) \mathbf{B}):(\mathbf{A}-\mathbf{B}) \geq 0 ;
$$

(ii) the function $\mu$ is continuous and

$$
0<\mu_{0}<\mu(s)<\mu_{1}, \quad \forall s \in \mathbb{R}_{+} ;
$$

(iii) $g \in L_{+}^{2}(\Omega)$, $\omega \in L_{+}^{2}\left(\Gamma_{0}\right)$, and $\mathbf{f} \in \mathbf{L}^{2}(\Omega)$;

(iv) the 2-dimensional Lebesgue measure of the set $\Gamma \backslash \Gamma_{0}$ is positive.

Remark 3. We claim that condition (i) holds true if the function $\mu$ is monotonically increasing. Indeed, using the Cauchy-Schwarz inequality, we obtain

$$
\begin{aligned}
(\mu & (|\mathbf{A}|) \mathbf{A}-\mu(|\mathbf{B}|) \mathbf{B}):(\mathbf{A}-\mathbf{B}) \\
= & \mu(|\mathbf{A}|)|\mathbf{A}|^{2}-\mu(|\mathbf{A}|) \mathbf{A}: \mathbf{B}-\mu(|\mathbf{B}|) \mathbf{B}: \mathbf{A} \\
& +\mu(|\mathbf{B}|)|\mathbf{B}|^{2} \\
\geq & \mu(|\mathbf{A}|)|\mathbf{A}|^{2}-\mu(|\mathbf{A}|)|\mathbf{A}||\mathbf{B}|-\mu(|\mathbf{B}|)|\mathbf{A}||\mathbf{B}| \\
& +\mu(|\mathbf{B}|)|\mathbf{B}|^{2} \\
= & \mu(|\mathbf{A}|)|\mathbf{A}|\{|\mathbf{A}|-|\mathbf{B}|\}-\mu(|\mathbf{B}|)|\mathbf{B}|\{|\mathbf{A}|-|\mathbf{B}|\} \\
= & \{\mu(|\mathbf{A}|)|\mathbf{A}|-\mu(|\mathbf{B}|)|\mathbf{B}|\}\{|\mathbf{A}|-|\mathbf{B}|\} \geq 0
\end{aligned}
$$

for any $\mathbf{A}, \mathbf{B} \in \mathbb{M}_{\text {sym }}^{3 \times 3}$.

\section{Weak Formulation of Problem (1)-(9)}

Definition 4 . One shall say that a vector function $\mathbf{u}: \bar{\Omega} \rightarrow \mathbb{R}^{3}$ is a weak solution to problem (1)-(9) if $\mathbf{u} \in \mathbf{X}(\Omega)$ and the following inequality holds:

$$
\begin{aligned}
& -\int_{\Omega}(\mathbf{u} \otimes \mathbf{u}): \mathbf{D}(\mathbf{v}) d x \\
& \quad+\int_{\Omega} \mu(|\mathbf{D}(\mathbf{u})|) \mathbf{D}(\mathbf{u}): \mathbf{D}(\mathbf{v}-\mathbf{u}) d x \\
& \quad+\int_{\Omega} g|\mathbf{D}(\mathbf{v})| d x+\int_{\Gamma_{0}} \omega|\mathbf{v}| d \Gamma \\
& \quad-\int_{\Omega} g|\mathbf{D}(\mathbf{u})| d x-\int_{\Gamma_{0}} \omega|\mathbf{u}| d \Gamma \\
& \geq \int_{\Omega} \mathbf{f} \cdot(\mathbf{v}-\mathbf{u}) d x
\end{aligned}
$$

for any vector function $\mathbf{v} \in \mathbf{X}(\Omega)$.
Remark 5. Let us explain how variational inequality (26) arises in the definition of weak solutions. Assume that regular functions $\mathbf{u}, \boldsymbol{\sigma}, \pi$ satisfy relations (1)-(9) and $\mathbf{v} \in \mathbf{X}(\Omega)$. If we take the scalar product of both sides of (1) by $\mathbf{v}-\mathbf{u}$ and integrate by parts over the domain $\Omega$, we get

$$
\begin{array}{r}
-\int_{\Omega}(\mathbf{u} \otimes \mathbf{u}): \mathbf{D}(\mathbf{v}) d x+\int_{\Omega} \boldsymbol{\sigma}: \mathbf{D}(\mathbf{v}-\mathbf{u}) d x \\
-\int_{\Gamma_{0}}(\boldsymbol{\sigma} \mathbf{n}) \cdot(\mathbf{v}-\mathbf{u}) d \Gamma=\int_{\Omega} \mathbf{f} \cdot(\mathbf{v}-\mathbf{u}) d x,
\end{array}
$$

where we used the equalities

$$
\int_{\Omega}(\mathbf{u} \otimes \mathbf{u}): \mathbf{D}(\mathbf{u}) d x=0,\left.\quad(\mathbf{u} \otimes \mathbf{u}) \mathbf{n}\right|_{\Gamma}=\mathbf{0} .
$$

Let us show that under conditions (3) and (4) the following inequality

$$
\begin{aligned}
\int_{\Omega} \boldsymbol{\sigma} & : \mathbf{D}(\mathbf{v}-\mathbf{u}) d x \\
\leq & \int_{\Omega} \mu(|\mathbf{D}(\mathbf{u})|) \mathbf{D}(\mathbf{u}): \mathbf{D}(\mathbf{v}-\mathbf{u}) d x \\
& \quad+\int_{\Omega} g|\mathbf{D}(\mathbf{v})| d x-\int_{\Omega} g|\mathbf{D}(\mathbf{u})| d x
\end{aligned}
$$

holds true. We set

$$
\begin{aligned}
& \Omega_{+}:=\{\mathbf{x} \in \Omega:|\mathbf{D}(\mathbf{u})(\mathbf{x})|>0\}, \\
& \Omega_{0}:=\{\mathbf{x} \in \Omega:|\mathbf{D}(\mathbf{u})(\mathbf{x})|=0\} .
\end{aligned}
$$

Using (3) and the Cauchy-Schwarz inequality, we obtain

$$
\begin{aligned}
& \int_{\Omega_{+}} \boldsymbol{\sigma}: \mathbf{D}(\mathbf{v}-\mathbf{u}) d x \\
& =\int_{\Omega_{+}}\left(\mu(|\mathbf{D}(\mathbf{u})|) \mathbf{D}(\mathbf{u})+g \frac{\mathbf{D}(\mathbf{u})}{|\mathbf{D}(\mathbf{u})|}\right): \mathbf{D}(\mathbf{v}-\mathbf{u}) d x \\
& =\int_{\Omega_{+}} \mu(|\mathbf{D}(\mathbf{u})|) \mathbf{D}(\mathbf{u}): \mathbf{D}(\mathbf{v}-\mathbf{u}) d x \\
& \quad+\int_{\Omega_{+}} g \frac{\mathbf{D}(\mathbf{u})}{|\mathbf{D}(\mathbf{u})|}: \mathbf{D}(\mathbf{v}) d x-\int_{\Omega_{+}} g|\mathbf{D}(\mathbf{u})| d x \\
& \leq \int_{\Omega_{+}} \mu(|\mathbf{D}(\mathbf{u})|) \mathbf{D}(\mathbf{u}): \mathbf{D}(\mathbf{v}-\mathbf{u}) d x \\
& \quad+\int_{\Omega_{+}} g|\mathbf{D}(\mathbf{v})| d x-\int_{\Omega_{+}} g|\mathbf{D}(\mathbf{u})| d x \\
& =\int_{\Omega} \mu(|\mathbf{D}(\mathbf{u})|) \mathbf{D}(\mathbf{u}): \mathbf{D}(\mathbf{v}-\mathbf{u}) d x \\
& \quad+\int_{\Omega_{+}} g|\mathbf{D}(\mathbf{v})| d x-\int_{\Omega} g|\mathbf{D}(\mathbf{u})| d x .
\end{aligned}
$$


Besides, taking into account (4), we arrive at the inequality

$$
\begin{aligned}
\int_{\Omega_{0}} \boldsymbol{\sigma}: \mathbf{D}(\mathbf{v}-\mathbf{u}) d x & =\int_{\Omega_{0}} \boldsymbol{\sigma}: \mathbf{D}(\mathbf{v}) d x \\
& \leq \int_{\Omega_{0}}|\boldsymbol{\sigma}||\mathbf{D}(\mathbf{v})| d x \\
& \leq \int_{\Omega_{0}} g|\mathbf{D}(\mathbf{v})| d x
\end{aligned}
$$

By adding this inequality to (31), we obtain relation (29).

Note also that the system of conditions (6)-(8) is equivalent to the following system:

$$
\begin{aligned}
& \left|(\boldsymbol{\sigma} \mathbf{n})_{\tan }\right| \leq \omega \text { on } \Gamma_{0}, \\
& (\boldsymbol{\sigma n})_{\tan } \cdot \mathbf{u}_{\tan }+\omega\left|\mathbf{u}_{\tan }\right|=0 \text { on } \Gamma_{0} \text {. }
\end{aligned}
$$

Using these relations, we obtain

$$
\begin{aligned}
& -\int_{\Gamma_{0}}(\boldsymbol{\sigma n}) \cdot(\mathbf{v}-\mathbf{u}) d \Gamma=-\int_{\Gamma_{0}}(\boldsymbol{\sigma} \mathbf{n})_{\tan } \cdot(\mathbf{v}-\mathbf{u})_{\tan } d \Gamma \\
& =-\int_{\Gamma_{0}}\left\{(\boldsymbol{\sigma} \mathbf{n})_{\tan } \cdot \mathbf{v}_{\tan }+\omega\left|\mathbf{u}_{\tan }\right|\right\} d \Gamma \\
& \quad \leq \int_{\Gamma_{0}} \omega|\mathbf{v}| d \Gamma-\int_{\Gamma_{0}} \omega|\mathbf{u}| d \Gamma .
\end{aligned}
$$

Finally, combining (27) with (29) and (34), we arrive at inequality (26).

\section{Main Results}

Our main results are collected in the following theorem.

Theorem 6. Suppose that conditions (i)-(iv) hold. Then

(a) problem (1)-(9) has at least one weak solution;

(b) any weak solution $\mathbf{u}$ satisfies the energy equality

$$
\begin{gathered}
\int_{\Omega} \mu(|\mathbf{D}(\mathbf{u})|)|\mathbf{D}(\mathbf{u})|^{2} d x+\int_{\Omega} g|\mathbf{D}(\mathbf{u})| d x \\
+\int_{\Gamma_{0}} \omega|\mathbf{u}| d \Gamma=\int_{\Omega} \mathbf{f} \cdot \mathbf{u} d x ;
\end{gathered}
$$

(c) the set of weak solutions to problem (1)-(9) is sequentially weakly closed in the space $\mathrm{X}(\Omega)$.

\section{Proof of Theorem 6}

The proof uses the following two propositions.

Proposition 7 (see [28]). Let $\mathbf{V}$ be a reflexive Banach space, $\mathbf{V}^{*}$ its the dual space, $\mathbf{A}: \mathbf{V} \rightarrow \mathbf{V}^{*}$ apseudomonotone operator, and $J: \mathbf{V} \rightarrow \mathbb{R}$ a lower semicontinuous convex functional. In addition, suppose that

$$
\frac{\langle\mathbf{A}(\mathbf{v}), \mathbf{v}\rangle+J(\mathbf{v})}{\|\mathbf{v}\|_{\mathbf{v}}} \longrightarrow+\infty
$$

as $\|\mathbf{v}\|_{\mathbf{V}} \rightarrow+\infty$. Then, for an arbitrary $\mathbf{z} \in \mathbf{V}^{*}$, there exists an element $\mathbf{u}_{\mathbf{z}} \in \mathbf{V}$ such that

$$
\left\langle\mathbf{A}\left(\mathbf{u}_{\mathbf{z}}\right)-\mathbf{z}, \mathbf{v}-\mathbf{u}_{\mathbf{z}}\right\rangle+J(\mathbf{v})-J\left(\mathbf{u}_{\mathbf{z}}\right) \geq 0 \quad \forall \mathbf{v} \in \mathbf{V} .
$$

Proposition 8 (Krasnoselskii's theorem, see [26]). Let $h: \Omega \times$ $\mathbb{R}^{m} \rightarrow \mathbb{R}$ be a function such that

(a) the function $h(\cdot, \mathbf{y}): \Omega \rightarrow \mathbb{R}$ is measurable for every $\mathbf{y} \in \mathbb{R}^{m}$;

(b) the function $h(\mathbf{x}, \cdot): \mathbb{R}^{m} \rightarrow \mathbb{R}$ is continuous for almost every $\mathbf{x} \in \Omega$;

(c) for every $\mathbf{y} \in \mathbb{R}^{m}$ and for almost every $\mathbf{x} \in \Omega$

$$
|h(\mathbf{x}, \mathbf{y})| \leq \alpha(\mathbf{x})+C \sum_{k=1}^{m}\left|y_{k}\right|^{p_{k} / q},
$$

where $p_{k}, q \geq 1, \alpha \in L^{q}(\Omega)$, and $C$ is a positive constant.

Then the Nemytskii operator defined by

$$
\begin{aligned}
& N_{h}: L^{p_{1}}(\Omega) \times \cdots \times L^{p_{m}}(\Omega) \longrightarrow L^{q}(\Omega), \\
& N_{h}\left[y_{1}, \ldots, y_{m}\right](\mathbf{x}):=h\left(\mathbf{x}, y_{1}(\mathbf{x}), \ldots, y_{m}(\mathbf{x})\right)
\end{aligned}
$$

is a bounded and continuous map.

Proof of Theorem 6. Let us introduce here the following operators:

$$
\begin{aligned}
\mathbf{A}_{\mu}: \mathbf{X}(\Omega) & \longrightarrow[\mathbf{X}(\Omega)]^{*}, \\
\left\langle\mathbf{A}_{\mu}(\mathbf{u}), \mathbf{v}\right\rangle & :=\int_{\Omega} \mu(|\mathbf{D}(\mathbf{u})|) \mathbf{D}(\mathbf{u}): \mathbf{D}(\mathbf{v}) d x, \\
\mathbf{K}_{f}: \mathbf{X}(\Omega) & \longrightarrow[\mathbf{X}(\Omega)]^{*}, \\
\left\langle\mathbf{K}_{f}(\mathbf{u}), \mathbf{v}\right\rangle: & =-\int_{\Omega}(\mathbf{u} \otimes \mathbf{u}): \mathbf{D}(\mathbf{v}) d x-\int_{\Omega} \mathbf{f} \cdot \mathbf{v} d x, \\
J_{g, \omega}: \mathbf{X}(\Omega) & \longrightarrow \mathbb{R}, \\
J_{g, \omega}(\mathbf{u}) & :=\int_{\Omega} g|\mathbf{D}(\mathbf{u})| d x+\int_{\Gamma_{0}} \omega|\mathbf{u}| d \Gamma .
\end{aligned}
$$

Using these operators, we can rewrite inequality (26) as follows:

$$
\left\langle\mathbf{A}_{\mu}(\mathbf{u})+\mathbf{K}_{f}(\mathbf{u}), \mathbf{v}-\mathbf{u}\right\rangle+J_{g, \omega}(\mathbf{v})-J_{g, \omega}(\mathbf{u}) \geq 0
$$

$\forall \mathbf{v} \in \mathbf{X}(\Omega)$.

By condition (i), we deduce that

$$
\left\langle\mathbf{A}_{\mu}(\mathbf{u})-\mathbf{A}_{\mu}(\mathbf{v}), \mathbf{u}-\mathbf{v}\right\rangle \geq 0 \quad \forall \mathbf{u}, \mathbf{v} \in \mathbf{X}(\Omega) ;
$$

that is, the operator $\mathbf{A}_{\mu}$ is monotone. Moreover, applying Proposition 8 and condition (ii), we establish that this operator is continuous. From properties of monotone operators it follows that $\mathbf{A}_{\mu}$ is a pseudomonotone operator. 
The embedding $\mathbf{H}^{1}(\Omega) \hookrightarrow \mathbf{L}^{4}(\Omega)$ is compact (see, e.g., [29]). This implies that the embedding $\mathbf{X}(\Omega) \hookrightarrow \mathbf{L}^{4}(\Omega)$ is compact too. Therefore, it is easily shown that the operator $\mathbf{K}_{f}$ is completely continuous; that is, if $\mathbf{u}_{n} \rightarrow \mathbf{u}_{0}$ weakly in the space $\mathbf{X}(\Omega)$ as $n \rightarrow \infty$, then $\mathbf{K}_{f}\left(\mathbf{u}_{n}\right) \rightarrow \mathbf{K}_{f}\left(\mathbf{u}_{0}\right)$ strongly in the space $[\mathbf{X}(\Omega)]^{*}$ as $n \rightarrow \infty$. This yields that the sum $\mathbf{A}_{\mu}+\mathbf{K}_{f}$ is a pseudomonotone operator. ity

Further, taking into account condition (ii) and the equal-

$$
\int_{\Omega}(\mathbf{u} \otimes \mathbf{u}): \mathbf{D}(\mathbf{u}) d x=0
$$

we obtain

$$
\frac{\left\langle\mathbf{A}_{\mu}(\mathbf{u})+\mathbf{K}_{f}(\mathbf{u}), \mathbf{u}\right\rangle+J_{g, \omega}(\mathbf{u})}{\|\mathbf{u}\|_{\mathbf{X}(\Omega)}} \longrightarrow+\infty
$$

as $\|\mathbf{u}\|_{\mathbf{X}(\Omega)} \rightarrow+\infty$.

Then from Proposition 7 we infer that inequality (41) has a solution $\mathbf{u}_{*} \in \mathbf{X}(\Omega)$. It is clear that $\mathbf{u}_{*}$ is a weak solution to problem (1)-(9).

We claim that energy equality (35) holds true for any weak solution $\mathbf{u}$ of problem (1)-(9). Indeed, by setting $\mathbf{v}=2 \mathbf{u}$ in (26), we find

$$
\begin{gathered}
\int_{\Omega} \mu(|\mathbf{D}(\mathbf{u})|)|\mathbf{D}(\mathbf{u})|^{2} d x+\int_{\Omega} g|\mathbf{D}(\mathbf{u})| d x \\
+\int_{\Gamma_{0}} \omega|\mathbf{u}| d \Gamma \geq \int_{\Omega} \mathbf{f} \cdot \mathbf{u} d x .
\end{gathered}
$$

On the other hand, the choice $\mathbf{v}=\mathbf{0}$ in (26) yields that

$$
\begin{gathered}
-\int_{\Omega} \mu(|\mathbf{D}(\mathbf{u})|)|\mathbf{D}(\mathbf{u})|^{2} d x-\int_{\Omega} g|\mathbf{D}(\mathbf{u})| d x \\
-\int_{\Gamma_{0}} \omega|\mathbf{u}| d \Gamma \geq-\int_{\Omega} \mathbf{f} \cdot \mathbf{u} d x .
\end{gathered}
$$

Obviously, if we combine the last inequality with (45), we get (35).

Now we must only prove that the set of weak solutions to problem (1)-(9) is sequentially weakly closed in the space $\mathbf{X}(\Omega)$. Consider a sequence $\left\{\mathbf{u}_{n}\right\}_{n=1}^{\infty}$ such that, for any $n \in \mathbb{N}$, $\mathbf{u}_{n}$ is a weak solution of (1)-(9) and $\mathbf{u}_{n} \rightarrow \mathbf{u}_{0}$ weakly in $\mathbf{X}(\Omega)$ as $n \rightarrow \infty$. Let us show that $\mathbf{u}_{0}$ is a weak solution of (1)-(9).

By definition of weak solutions, we have

$$
\begin{aligned}
& \left\langle\mathbf{A}_{\mu}\left(\mathbf{u}_{n}\right)+\mathbf{K}_{f}\left(\mathbf{u}_{n}\right), \mathbf{v}-\mathbf{u}_{n}\right\rangle+J_{g, \omega}(\mathbf{v})-J_{g, \omega}\left(\mathbf{u}_{n}\right) \\
& \geq 0 \quad \forall \mathbf{v} \in \mathbf{X}(\Omega), n \in \mathbb{N} .
\end{aligned}
$$

Note that the functional $J_{g, \omega}: \mathbf{X}(\Omega) \rightarrow \mathbb{R}$ is convex and continuous. Therefore, $J_{g, w}$ is lower semicontinuous with respect to the weak convergence in $\mathbf{X}(\Omega)$. This implies that

$$
J_{g, \omega}\left(\mathbf{u}_{0}\right) \leq \liminf _{n \rightarrow \infty} J_{g, \omega}\left(\mathbf{u}_{n}\right) .
$$

Further, we set $\mathbf{v}=\mathbf{u}_{0}$ in (47) and pass to the lower limit as $n \rightarrow \infty$. Taking into account inequality (48) and the complete continuity of the operator $\mathbf{K}_{f}$, we obtain

$$
\liminf _{n \rightarrow \infty}\left\langle\mathbf{A}_{\mu}\left(\mathbf{u}_{n}\right), \mathbf{u}_{0}-\mathbf{u}_{n}\right\rangle \geq 0,
$$

or equivalently,

$$
\limsup _{n \rightarrow \infty}\left\langle\mathbf{A}_{\mu}\left(\mathbf{u}_{n}\right), \mathbf{u}_{n}-\mathbf{u}_{0}\right\rangle \leq 0 .
$$

Since $\mathbf{A}_{\mu}$ is a pseudomonotone operator, it follows from the last inequality that

$$
\begin{aligned}
& \liminf _{n \rightarrow \infty}\left\langle\mathbf{A}_{\mu}\left(\mathbf{u}_{n}\right), \mathbf{u}_{n}-\mathbf{v}\right\rangle \geq\left\langle\mathbf{A}_{\mu}\left(\mathbf{u}_{0}\right), \mathbf{u}_{0}-\mathbf{v}\right\rangle \\
& \forall \mathbf{v} \in \mathbf{X}(\Omega) .
\end{aligned}
$$

Now, we rewrite (47) in the form

$$
\begin{array}{r}
J_{g, \omega}\left(\mathbf{u}_{n}\right)-J_{g, \omega}(\mathbf{v}) \leq-\left\langle\mathbf{A}_{\mu}\left(\mathbf{u}_{n}\right)+\mathbf{K}_{f}\left(\mathbf{u}_{n}\right), \mathbf{u}_{n}-\mathbf{v}\right\rangle \\
\forall \mathbf{v} \in \mathbf{X}(\Omega), n \in \mathbb{N}
\end{array}
$$

and pass to the upper limit in this inequality:

$$
\begin{aligned}
& \limsup _{n \rightarrow \infty}\left\{J_{g, \omega}\left(\mathbf{u}_{n}\right)-J_{g, \omega}(\mathbf{v})\right\} \\
& \quad \leq \limsup _{n \rightarrow \infty}\left\{-\left\langle\mathbf{A}_{\mu}\left(\mathbf{u}_{n}\right)+\mathbf{K}_{f}\left(\mathbf{u}_{n}\right), \mathbf{u}_{n}-\mathbf{v}\right\rangle\right\} .
\end{aligned}
$$

Using (48) and (51), we deduce from (53) that

$$
\begin{aligned}
& J_{g, \omega}\left(\mathbf{u}_{0}\right)-J_{g, \omega}(\mathbf{v}) \leq \liminf _{n \rightarrow \infty}\left\{J_{g, \omega}\left(\mathbf{u}_{n}\right)-J_{g, \omega}(\mathbf{v})\right\} \\
& \quad \leq \limsup _{n \rightarrow \infty}\left\{J_{g, \omega}\left(\mathbf{u}_{n}\right)-J_{g, \omega}(\mathbf{v})\right\} \\
& \quad \leq \limsup _{n \rightarrow \infty}\left\{-\left\langle\mathbf{A}_{\mu}\left(\mathbf{u}_{n}\right)+\mathbf{K}_{f}\left(\mathbf{u}_{n}\right), \mathbf{u}_{n}-\mathbf{v}\right\rangle\right\} \\
& =-\liminf _{n \rightarrow \infty}\left\{\left\langle\mathbf{A}_{\mu}\left(\mathbf{u}_{n}\right)+\mathbf{K}_{f}\left(\mathbf{u}_{n}\right), \mathbf{u}_{n}-\mathbf{v}\right\rangle\right\} \\
& \leq-\left\langle\mathbf{A}_{\mu}\left(\mathbf{u}_{0}\right)+\mathbf{K}_{f}\left(\mathbf{u}_{0}\right), \mathbf{u}_{0}-\mathbf{v}\right\rangle \\
& =\left\langle\mathbf{A}_{\mu}\left(\mathbf{u}_{0}\right)+\mathbf{K}_{f}\left(\mathbf{u}_{0}\right), \mathbf{v}-\mathbf{u}_{0}\right\rangle \quad \forall \mathbf{v} \in \mathbf{X}(\Omega) .
\end{aligned}
$$

Thus, we have

$$
\begin{array}{r}
\left\langle\mathbf{A}_{\mu}\left(\mathbf{u}_{0}\right)+\mathbf{K}_{f}\left(\mathbf{u}_{0}\right), \mathbf{v}-\mathbf{u}_{0}\right\rangle+J_{g, \omega}(\mathbf{v})-J_{g, \omega}\left(\mathbf{u}_{0}\right) \geq 0 \\
\forall \mathbf{v} \in \mathbf{X}(\Omega) .
\end{array}
$$

This means that $\mathbf{u}_{0}$ is a weak solution of problem (1)-(9).

Theorem 6 is completely proved.

\section{Conflicts of Interest}

The author declares that there are no conflicts of interest regarding the publication of this paper.

\section{Acknowledgments}

This work was supported by the Russian Foundation for Basic Research, Project no. 16-31-00182 mol_a. 


\section{References}

[1] K. R. Rajagopal, "On some unresolved issues in non-linear fluid dynamics," Russian Mathematical Surveys, vol. 58, no. 2, pp. 319330, 2003.

[2] E. C. Bingham, "An investigation of the laws of plastic flow," Bulletin of the Bureau of Standards, vol. 13, no. 2, pp. 309-353, 1916.

[3] E. C. Bingham, Fluidity and Plasticity, McGraw Hill, New York, NY, USA, 1922.

[4] H. Fujita, "A mathematical analysis of motions of viscous incompressible fluid under leak or slip boundary conditions," RIMS Kokyuroku, vol. 888, no. 1, pp. 199-216, 1994.

[5] C. Le Roux and A. Tani, "Steady solutions of the NavierStokes equations with threshold slip boundary conditions," Mathematical Methods in the Applied Sciences, vol. 30, no. 5, pp. 595-624, 2007.

[6] P. P. Mosolov and V. P. Miasnikov, "Variational methods in the theory of the fluidity of a viscous-plastic medium," Journal of Applied Mathematics and Mechanics, vol. 29, no. 3, pp. 545-577, 1965.

[7] G. Duvaut and J.-L. Lions, Inequalities in Mechanics and Physics, Springer, Berlin, Germany, 1976.

[8] J. U. Kim, "On the Cauchy problem associated with the motion of a Bingham fluid in the plane," Transactions of the American Mathematical Society, vol. 298, no. 1, pp. 371-400, 1986.

[9] J. U. Kim, "On the initial-boundary value problem for a Bingham fluid in a three-dimensional domain," Transactions of the American Mathematical Society, vol. 304, no. 2, pp. 751-770, 1987.

[10] G. A. Seregin, "On a dynamical system generated by the twodimensional equations of the motion of a Bingham fluid," Journal of Mathematical Sciences, vol. 70, no. 3, pp. 1806-1816, 1994.

[11] V. V. Shelukhin, "Bingham viscoplastic as a limit of nonNewtonian fluids," Journal of Mathematical Fluid Mechanics, vol. 4, no. 2, pp. 109-127, 2002.

[12] J. Malek, M. Ruzicka, and V. V. Shelukhin, "Herschel-Bulkley fluids: existence and regularity of steady flows," Mathematical Models and Methods in Applied Sciences, vol. 15, no. 12, pp. 18451861, 2005.

[13] A. E. Mamontov, "Existence of global solutions to multidimensional equations for Bingham fluids," Mathematical Notes, vol. 82, no. 3-4, pp. 501-517, 2007.

[14] H. Eberlein and M. Ruzicka, "Existence of weak solutions for unsteady motions of Herschel-Bulkley fluids," Journal of Mathematical Fluid Mechanics, vol. 14, no. 3, pp. 485-500, 2012.

[15] Y. Amirat and V. V. Shelukhin, "Nonhomogeneous incompressible Herschel-Bulkley fluid flows between two eccentric cylinders," Journal of Mathematical Fluid Mechanics, vol. 15, no. 4, pp. 635-661, 2013.

[16] L. Fusi, A. Farina, and F. Rosso, "Bingham flows with pressuredependent rheological parameters," International Journal of Non-Linear Mechanics, vol. 64, pp. 33-38, 2014.

[17] N. El Khouja, N. Roquet, and B. Cazacliu, "Analysis of a regularized Bingham model with pressure-dependent yield stress," Journal of Mathematical Fluid Mechanics, vol. 17, no. 4, pp. 723-739, 2015.

[18] V. V. Shelukhin and V. V. Neverov, "Thermodynamics of micropolar Bingham fluids," Journal of Non-Newtonian Fluid Mechanics, vol. 236, pp. 83-90, 2016.
[19] L. Fusi and A. Farina, "Flow of a Bingham fluid in a non symmetric inclined channel," Journal of Non-Newtonian Fluid Mechanics, vol. 238, pp. 24-32, 2016.

[20] M. Ruzicka, V. Shelukhin, and M. M. dos Santos, "Steady flows of Cosserat-Bingham fluids," Mathematical Methods in the Applied Sciences, vol. 40, no. 7, pp. 2746-2761, 2017.

[21] R. Mahmood, N. Kousar, M. Yaqub, and K. Jabeen, "Numerical simulations of the square lid driven cavity flow of Bingham fluids using nonconforming finite elements coupled with a direct solver," Advances in Mathematical Physics, vol. 2017, Article ID 5210708, 10 pages, 2017.

[22] Y. Damianou and G. C. Georgiou, "On Poiseuille flows of a Bingham plastic with pressure-dependent rheological parameters," Journal of Non-Newtonian Fluid Mechanics, vol. 250, pp. $1-7,2017$.

[23] L. Fusi, "Non-isothermal flow of a Bingham fluid with pressure and temperature dependent viscosity," Meccanica, vol. 52, pp. 3577-3592, 2017.

[24] L. Consiglieri, "A nonlocal friction problem for a class of nonNewtonian flows," Portugaliae Mathematica, vol. 60, no. 2, pp. 237-252, 2003.

[25] M. Yu. Kuzmin, On boundary-value problems for some models of fluids dynamics with slip boundary conditions [Candidates dissertation in Mathematics and Physics], Voronezh State University, Voronezh, Russia, 2007.

[26] M. A. Krasnoselskii, Topological Methods in the Theory of Nonlinear Integral Equations, Gostehizdat, Moscow, Russia, 1956.

[27] V. G. Litvinov, Motion of a Nonlinear-Viscous Fluid, Nauka, Moscow, Russia, 1982.

[28] J. L. Lions, Quelques Methodes de Resolution des Problemes aux Limites non Lineaires, Dunod Gauthier-Villars, Paris, France, 1969.

[29] R. A. Adams and J. J. F. Fournier, Sobolev Spaces, vol. 40 of Pure and Applied Mathematics, Academic Press, Amsterdam, the Netherlands, 2003. 


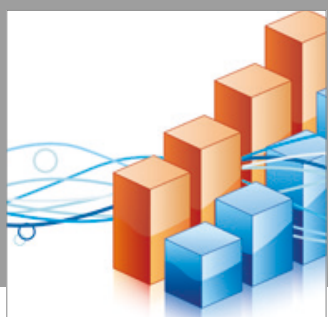

Advances in

Operations Research

vatersals

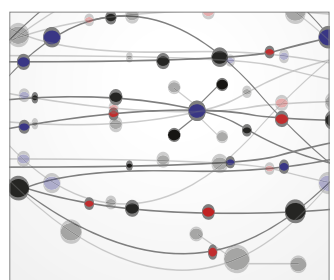

\section{The Scientific} World Journal
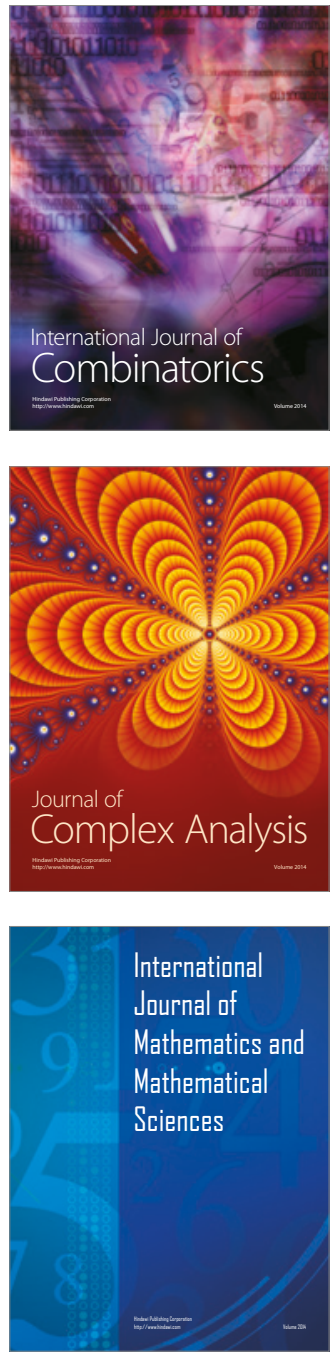
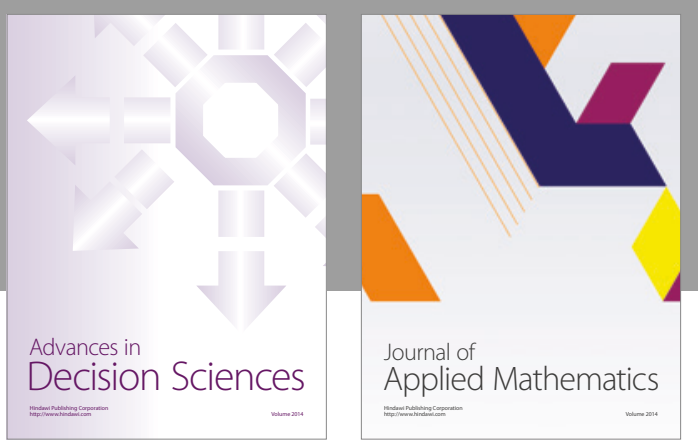

Algebra

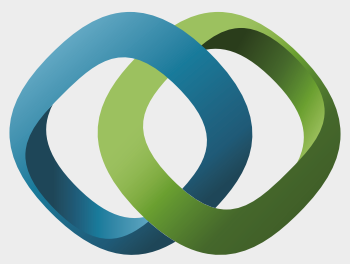

\section{Hindawi}

Submit your manuscripts at

https://www.hindawi.com
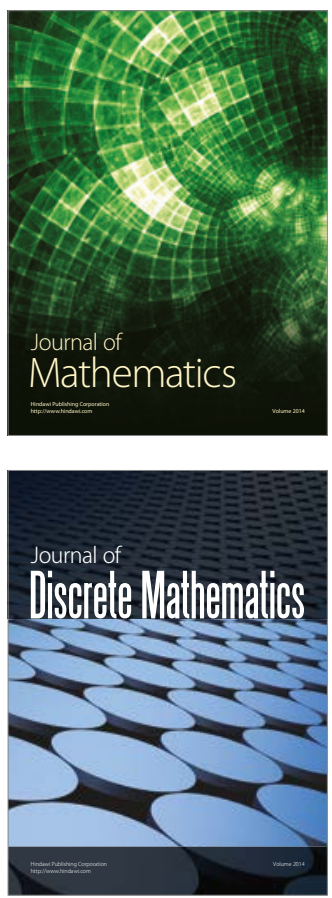

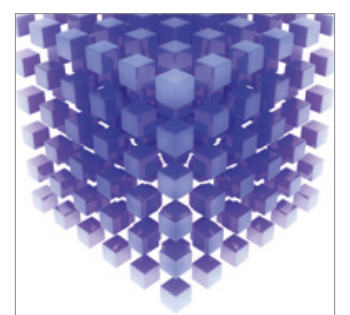

Mathematical Problems in Engineering
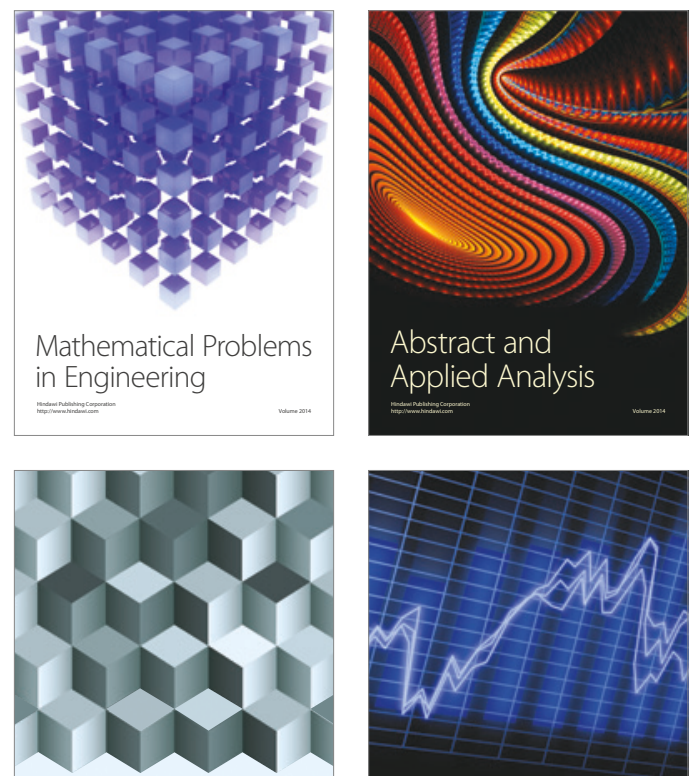

Journal of

Function Spaces

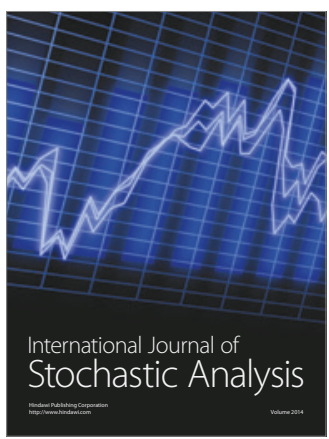

Probability and Statistics
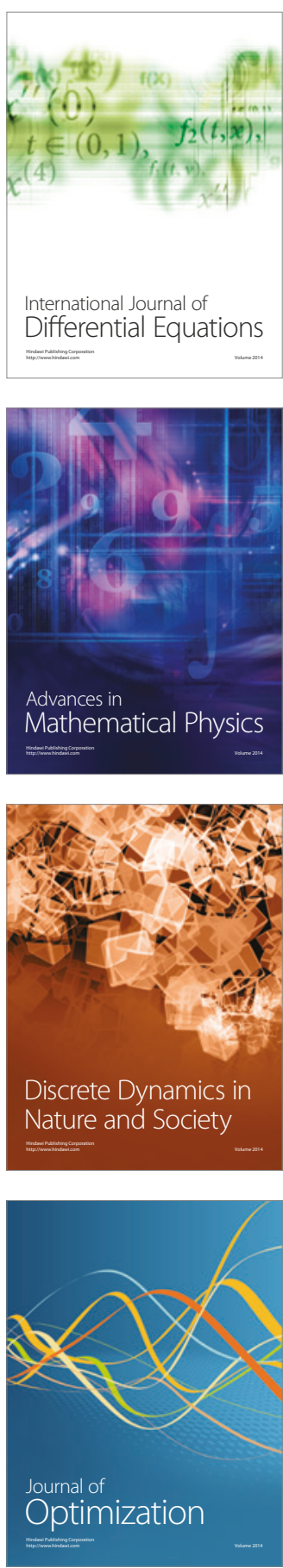